\title{
Effect of high magnetic field on phase and structural transformations in ferromagnetic alloys. Review
}

\author{
V. A. Milyutin ${ }^{\dagger}$ I. V. Gervasyeva \\ †milutin@imp.uran.ru
}

Institute of metal physics UB RAS, 18 S. Kovalevskaya str., Yekaterinburg, 620990, Russia

Review of relevant research about the effect of high DC magnetic field on structural and phase transformations in different ferromagnetic alloys are presented. Main experimental results of simultaneous impact high temperature and magnetic field with induction up to $45 \mathrm{~T}$ on alloys $\mathrm{Fe}-\mathrm{C}, \mathrm{Fe}-\mathrm{Co}, \mathrm{Fe}-\mathrm{Si}, \mathrm{Mn}-\mathrm{Bi}, \mathrm{Fe}-\mathrm{Ni}, \mathrm{Ni}-\mathrm{Co}$ and other are presented. The main high magnetic field effect on a phase transformation is equilibrium temperature changing, which reaches tens degrees. Modern views about cause of such effect, which consist in changing of thermodynamic conditions of transformation under external magnetic field due different magnetic properties between parent and appearing phase are described. In the theoretical and experimental determination of the phase transformation temperature shift magnitude in most cases there is a significant discrepancy. The probable causes such discrepancy are discussed. Moreover, review of results on investigations effect of magnetic field on structure transformations; in particular recovery and recrystallization in cold-rolled ferromagnetic alloys are presented. It is shown, that magnetic field retards recovery process and furthermore promotes preferential formation of certain texture components. Nowadays there is no consensus in the scientific literature on the nature of the observed effects. In this paper, critical analyses of the approaches proposed by different authors to the interpretation of experimental results are presented. The current state of the problem is described; in conclusion, the prospects for further studies of thermally activated processes in the conditions of application of a high DC magnetic field are indicated.

Keywords: high magnetic field, ferromagnetic alloys, structure, crystallographic texture, structural and phase transformations.

УДК: 538.911

\section{Влияние сильного магнитного поля на фазовые и структурные превращения в ферромагнитных сплавах. Обзор}

\author{
Милютин В. А. ${ }^{\dagger}$, Гервасьева И. В. \\ Институт физики металлов УрО РАН, ул. С. Ковалевской, 18, Екатеринбург, 620990, Россия
}

Представлен обзор наиболее актуальных исследований, посвященных вопросу влияния сильного постоянного магнитного поля на структурные и фазовые превращения в различных ферромагнитных сплавах. Приводятся основные результаты экспериментов по одновременному воздействию повышенной температуры и магнитного поля с индукцией до 45 Тл на сплавы системы Fe-C, Fe-Co, Fe-Si, Mn-Bi, Fe-Ni, Ni-Co и некоторые другие. Основным эффектом, оказываемым сильным магнитным полем на фазовые превращения в указанных сплавах, является изменение температуры равновесия, которое достигает десятков градусов. Описаны современные представления о причине такого влияния, которая заключается в изменении термодинамических условий превращения во внешнем магнитном поле вследствие различия магнитных свойств исходной и формирующейся фаз. При теоретическом и экспериментальном определении величины смещения температуры фазового превращения в большинстве случаев наблюдается существенное расхождение. Обсуждаются возможные причины такого несоответствия. Помимо этого, приведен обзор результатов исследования влияния магнитного поля на структурные превращения, в частности процессы возврата и рекристаллизации в холоднокатаных ферромагнитных сплавах. Показано, что магнитное поле замедляет возврат, и, кроме того, способствует преимущественному формированию определенных текстурных компонент. На сегодняшний день в научной литературе нет единого мнения относительно природы наблюдаемых эффектов. В настоящей работе приводится критический анализ предложенных разными авторами подходов к интерпретации экспериментальных результатов. Описывается современное состояние проблемы, в заключении обозначены перспективы дальнейших исследований термоактивируемых процессов в условиях приложения сильного постоянного магнитного поля.

Ключевые слова: сильное магнитное поле, ферромагнитные сплавы, структура, кристаллографическая текстура, структурные и фазовые превращения. 


\section{1. Введение}

Магнитное поле, наряду с температурой и давлением, является одним из внешних факторов, за счет которых можно воздействовать на формирующуюся структуру металлического материала. Приложение магнитного поля во время термообработки металлов и сплавов с целью изменения их микроструктуры и свойств используется уже порядка ста лет. За это время было проведено большое количество как теоретических, так и экспериментальных исследований. Обнаружено много эффектов, вызванных приложением магнитного поля, таких как смещение температуры фазовых переходов, возникновение наведенной магнитной анизотропии, направленная кристаллизация из расплава, изменение скорости диффузии и т. д. Поскольку указанные эффекты отличаются друг от друга по своей природе, то отличаются и условия, при которых они возникают. Используются как слабые, так и сильные постоянные и переменные магнитные поля. В качестве материалов исследования выступают металлы и сплавы с разным типом магнитного упорядочения. В силу этих обстоятельств в научном сообществе ещё не в полной мере сформировались представления о роли обработки, включающей одновременное воздействие температуры и внешнего магнитного поля, на структуру и свойства материалов.

Часто под термомагнитной обработкой понимают нагрев и охлаждение материала в продольном или поперечном магнитном поле с целью воздействия на свойства. Наиболее действенной такая обработка является в магнитомягких сплавах на основе железа, имеющих широкое применение в электротехнической промышленности [1,2]. С помощью термомагнитной обработки в этих сплавах можно существенным образом изменять магнитные свойства. Основной причиной эффекта является возникновение индуцированной магнитной анизотропии вследствие упорядочения пар атомов определенного сорта в направлении прикладываемого поля [3]. Для того чтобы такая обработка была эффективна, требуется нагрев в область температур, достаточных для активизации диффузионного движения атомов, в условиях приложения магнитного поля. Широкое распространение она получила, в том числе, за счёт того, что для получения эффекта достаточно приложения слабых магнитных полей с индукцией < 1 Тл, которые легко создавать с помощью традиционных электромагнитов.

Однако, для внесения изменений в термодинамику превращения и инициирования других эффектов, вызванных магнитным полем, необходимы поля с гораздо большей индукцией. Деление магнитных полей на сильные и слабые, исходя из их величины, всегда носит условный характер, поскольку необходимая величина прикладываемого поля варьируется в зависимости от конкретных задач. Так, например, для того, чтобы оказать воздействие на движение электронов в атоме, необходимо приложение магнитного поля, порядка 100 Тл [4]. А при воздействии магнитного поля на термически активируемые процессы, магнитная энергия для магнитного момента $1 \mu \mathrm{B}$ в поле 1 Тл соответствует тепловой энергии при температуре 0,67 К [5]. В этой связи можно подразделить магнитные поля на слабые и сильные в зависимости от способа их получения. Традиционный электромагнит, состоящий из обмотки и магнитопровода, позволяет создать постоянное магнитное поле, с индукцией, как правило, не превышающей 3 Тл [6]. Постоянные магнитные поля с большей индукцией получают в магнитах Биттера, в магнитах со сверхпроводящими обмотками, либо в гибридных магнитах, такие поля мы и будем подразумевать под сильными. В последние два десятилетия получили распространение безгелиевые Биттеровские магниты, позволяющие проводить длительные высокотемпературные отжиги в сильном магнитном поле. Максимальная доступная на сегодняшний день величина постоянного магнитного поля составляет 45 Тл. Настоящая работа посвящена вопросам влияния именно сильного магнитного поля на фазовые и структурные превращения при нагреве.

В течение последнего десятилетия наблюдается рост публикационной активности в области исследования влияния постоянного магнитного поля на фазовые и структурные превращения, который связан, в первую очередь, с появлением нового оборудования. Появляются и обзоры таких исследований $[7,8]$. Необходимость поиска перспектив практического применения отжигов в сильном постоянном магнитном поле, а также изучения теоретических основ явлений, происходящих при формировании структуры в материале под действием внешнего поля, делает такие работы интересными и актуальными. Большое количество исследований по влиянию магнитного поля на превращения в материалах с разной кристаллической решеткой, разным типом магнитного упорядочения вызывают необходимость систематизировать имеющиеся результаты. Поэтому в настоящей статье рассматриваются работы, посвященные только превращениям в ферромагнитных материалах, в обзор включены как работы, имеющиеся в научной литературе, так и экспериментальные исследования авторов.

С точки зрения классификации эффектов, оказываемых сильным магнитным полем на термоактивируемые процессы, их можно разделить на две большие группы, а именно, эффекты, связанные с фазовыми превращениями, и эффекты, связанные со структурными превращениями при нагреве (возврат и рекристаллизация).

\section{2. Фазовые превращения в условиях магнитного поля}

Изучение влияния магнитного поля на фазовые переходы началось с работ по обнаружению эффектов, оказываемых сильным импульсным магнитным полем на сдвиговое мартенситное превращение [9-11], заключающихся в смещении точки начала превращения, изменении количественного соотношения фаз, а также изменения морфологии образующихся кристаллов мартенсита $[12,13]$. Этим исследованиям положили начало работы В. Д. Садовского и М. А. Кривоглаза $[14,15]$ в которых они предложили формулу для оценки смещения температуры мартенситного перехода в магнитном поле: 


$$
d T=T_{0}\left(V_{1} M_{1}-V_{2} M_{2}\right) H / q,
$$

где $M_{1}$ и $M_{2}$ - магнитные моменты фаз, участвующих в превращении; $H$ - напряженность магнитного поля; $V_{1}$ и $V_{2}$ - объемы первой и второй фаз, $T_{0}-$ температура фазового равновесия; $q$ - теплота превращения.

Позднее был выполнен ряд работ по уточнению уравнения Кривоглаза-Садовского путём учёта дополнительных параметров, например, магнитострикционных эффектов [16] и неоднородности магнитной структуры аустенитной фазы [17]. В общем случае, для большинства сплавов с неферромагнитным аустенитом смещение температуры начала мартенситного превращения в поле удовлетворительно описывается уравнением Кривоглаза-Садовского. Развитие исследований мартенситного превращения в магнитном поле с исторической точки зрения описано в работах $[18,19]$.

Мартенситное превращение, проходящее бездиффузионным путём, обладает высокой скоростью протекания, за счёт этого достаточно приложить импульсное поле с длительностью импульса порядка $10^{-4}$ с для внесения существенных изменений в термодинамику превращения. Для проведения аналогичных исследований в случае диффузинно-контролируемых фазовых превращений требуется длительное одновременное воздействие температуры и магнитного поля. Оборудование, позволяющее проводить такие эксперименты, стало доступным только в последние 15-20 лет, с его появлением связан рост числа публикаций, посвященных этому вопросу. В начале 2000-х годов появились теоретические работы, в которых производился расчёт термодинамических параметров фазового превращения в системе $\mathrm{Fe}-\mathrm{C}$ в условиях сильного магнитного поля [20,21]. В статье [21] авторы использовали молекулярную теорию Вейсса для расчетов и пришли к выводу, что сильное магнитное поле повышает температуру равновесия аустенит/феррит в сплаве $\mathrm{Fe}-\mathrm{C}$, а также увеличивает растворимость углерода в феррите. Впоследствии эти расчеты были подтверждены экспериментально [22,23].

С точки зрения термодинамических подходов сплавы на основе железа являются подходящим материалом для исследования влияния магнитного поля на фазовые превращения, поскольку $\alpha$ - и $\gamma$-фазы существенно различны по магнитным свойствам. Для сплавов на основе железа получена целая серия результатов по влиянию магнитного поля на фазовые превращения. Показано, что в общем случае приложение магнитного поля повышает температуры $\alpha \rightarrow \gamma$ и $\gamma \rightarrow \alpha$. Были исследованы сплавы Fe-C [24], Fe-Co [25], Fe-Si [26], Fe-Mn-C-Nb [27], и другие. C помощью метода CALPHAD на основании изменений вносимых внешним магнитным полем в энергию Гиббса показано, что в сплаве $\mathrm{Fe}-\mathrm{Si}$ существенно сужается $\gamma$-область при увеличении индукции прикладываемого поля [26]. В работе [24] были экспериментально определены температуры $\mathrm{A}_{c 1}$ и $\mathrm{A}_{c 3}$ в сплавах $\mathrm{Fe}-0.18$ (масс.\%) С и $\mathrm{Fe}-0.38$ (масс.\%) С. По этим данным авторы построили фрагмент диаграммы состояния $\mathrm{Fe}-\mathrm{C}$ в условиях наложения внешнего магнитного поля (Рис. 1).

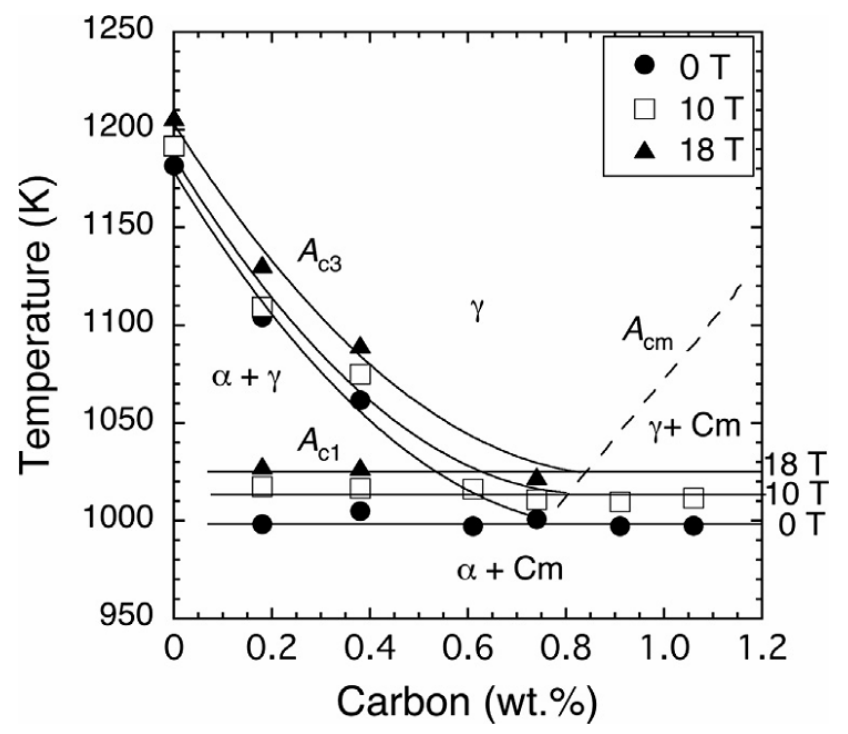

Рис. 1. Фрагмент фазовой диаграммы Fe-C в магнитном поле до 18 Тл. [24].

Fig. 1. Fragment of Fe-C phase diagram under magnetic field up to $18 \mathrm{~T}[24]$.

Очевидно, что величина смещения температуры фазового превращения коренным образом зависит от магнитного состояния формирующейся фазы при данной температуре. В данном случае увеличение температуры $\mathrm{A}_{c 3}$ пропорционально приблизительно квадрату величины магнитного поля, в то время как смещение $\mathrm{A}_{c 1}$ - линейно растет при увеличении внешнего поля. Причиной является тот факт, что при температурах превращения $\alpha+\gamma \rightarrow \gamma$ феррит (точка Кюри $1043 \mathrm{~K}$ ) парамагнитен и энергия Зеемана, которая вносит вклад в термодинамический потенциал, равна:

$$
E_{M}=1 / 2 \chi B^{2},
$$

где $\chi$ - парамагнитная восприимчивость, $B$ - индукция магнитного поля.

В ферромагнитном состоянии (температура $\mathrm{A}_{\mathrm{c1}}$ ) она равна

$$
E_{M}=\int_{0}^{B} M_{\alpha}(T, B) d B
$$

Аналогичные результаты получены на примере сплавов, в которых легирование приводит к тому, что соотношение

$$
T_{0} / T_{c},
$$

где $T_{0}$ - температура фазового равновесия, $T_{c}-$ точка Кюри, меняется от $<1$ к >1 или наоборот. Такими сплавами являются, например, Fe-Ni [29], Fe-Rh, Fe-Co.

$\mathrm{B}$ сплаве $\mathrm{Fe}-\mathrm{Co}$ при повышении содержания кобальта температура Кюри увеличивается более интенсивно, чем температура фазового равновесия (Табл. 1). Благодаря этому в сплавах $\mathrm{Fe}-20$ (ат.\%) Со и $\mathrm{Fe}-30$ (ат.\%) Co $\alpha$-фаза при температуре фазового превращения находится в ферромагнитном состоянии. На Рис. 2 представлены зависимости $\Delta T$ от магнитного поля в этих сплавах, полученные как экспериментально, так и теоретически. Можно видеть, что в чистом железе температура фазового перехода смещается на 7 градусов Кельвина, тогда как в сплаве $\mathrm{Fe}-30$ (ат.\%) Со на 24 [25]. 
Необходимо отдельно рассмотреть серию исследований выполненных на системе MnBi $[29,30]$. В данном интерметаллиде низкотемпературная фаза MnBi с гексагональной структурой типа NiAs обладает уникальными магнитными свойствами. Магнитный момент при $0 \mathrm{~K}$ составляет 3,9 $\mu \mathrm{B}$, рассчитанная температура Кюри 720 К. Однако, при увеличении температуры (630 K) происходит фазовый переход. Выделяется высокотемпературная фаза $\mathrm{Mn}_{1.08} \mathrm{Bi}$ с гексагональной структурой типа $\mathrm{Ni}_{2} \mathrm{In}$, магнитный момент которой составляет $1.7 \mu \mathrm{B}$, а температура Кюри $473 \mathrm{~K}$. В этой связи можно ожидать значительного эффекта, оказываемого магнитным полем на смещение температуры фазового превращения $\mathrm{MnBi} \rightarrow \mathrm{Mn}_{1,08} \mathrm{Bi}$. В работе [29] показано, что смещение в постоянном магнитном поле 45 Тл достигает 82 градусов Кельвина, что приблизительно равно 2 К/Тл (Рис. 3). При этом область существования высокотемпературной фазы $\mathrm{Mn}_{1,08}$ Ві сужается всего до 12 градусов.

Термодинамический подход хорошо описывает наблюдаемые во многих материалах явления, с качественной точки зрения. Недостатком является, то, что расчетные и экспериментальные величины смещения температуры не всегда хорошо согласуются между собой $[24,25,27]$. Даже учёт дополнительных термодинамических параметров, помимо вклада энергии Зеемана не даёт хорошего совпадения. Вероятно, для того чтобы точно предсказать поведение температуры фазового превращения в магнитном поле необходимо учитывать также ряд дополнительных параметров, таких как морфология частиц выделяющейся фазы, магнитострикционные явления, изменение скорости диффузии в магнитном поле о котором сообщается, например, в [31] и другие.

\section{3. Структурные превращения в условиях магнитного поля}

В работах [32,33] было показано, что магнитное поле может влиять не только на фазообразование, но и на структурные превращения, в частности, на рекристаллизацию холоднодеформированных металлических материалов. Такое влияние проявляется в изменении скорости протекания процессов рекристаллизации [33], а также в изменении микроструктуры и кристаллографической текстуры [34], подвергнутых магнитному отжигу образцов. В настоящий момент в научной литературе насчитывается не так много работ по исследованию структурных превращений в условиях наличия внешнего магнитного поля. Приводимые в немногочисленных статьях результаты экспериментов и их интерпретация часто противоречат друг другу. Авторы [35] подвергали отжигу в магнитном поле 10 Тл образцы из сплава $\mathrm{Fe}-3,25 \% \mathrm{Si}$ с текстурой деформации $\{111\}<112>$. При этом формировалась более острая текстура $\{110\}<001>$, чем при отжиге без поля. Это объясняли облегчением процессов протекания возврата и рекристаллизации в этих зернах за счёт наименьшей магнитострикции при совпадении осей <001> с полем. К аналогичному выводу пришли и авторы работы [32], которые проводили эксперименты по магнитному отжигу образцов из сплава $\mathrm{Fe}-35 \% \mathrm{Co}$.
Табл. 1. Скрытая теплота $(L)$, спонтанная намагниченность при $0 \mathrm{~K}$, Температура Кюри $T_{c}$ и температура равновесия при нулевом магнитном поле $T_{0}[25]$.

Table 1. Latent heat $L$, spontaneous magnetization at $0 \mathrm{~K}\left(M_{0}\right)$, Curie temperature $T_{c}$ and equilibrium temperature at zero field $T_{0}$ $(H=0)[25]$.

\begin{tabular}{|c|c|c|c|c|}
\hline $\begin{array}{c}\text { Сплав } \\
\text { Alloy }\end{array}$ & $L, \mathrm{~J} / \mathrm{mol}$ & $M_{0}$ & $T_{c}, \mathrm{~K}$ & $T_{0}(H=0)$ \\
\hline чистое Fe/pure Fe & 920 & 2.22 & 1043 & 1184.3 \\
$\mathrm{Fe}-10 \mathrm{Co}$ & 1730 & 2.32 & 1155 & 1180.1 \\
$\mathrm{Fe}-20 \mathrm{Co}$ & 3940 & 2.42 & 1267 & 1220.9 \\
$\mathrm{Fe}-30 \mathrm{Co}$ & 5100 & 2.46 & 1299 & 1236.2 \\
\hline
\end{tabular}

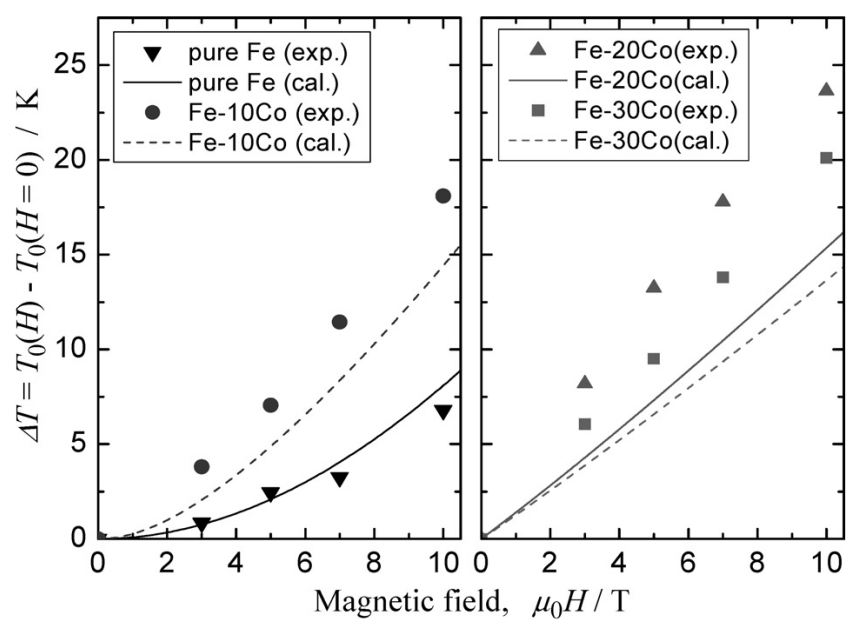

Рис. 2. Изменение температуры $\alpha-\gamma$ превращения в сплаве $\mathrm{Fe}-\mathrm{Co}$ с приложением магнитного поля. Расчетные и экспериментальные значения. [25].

Fig. 2. Shift of $\alpha-\gamma$ transformation temperature of Fe-Co alloy under magnetic field. Calculated and experimental results [25].

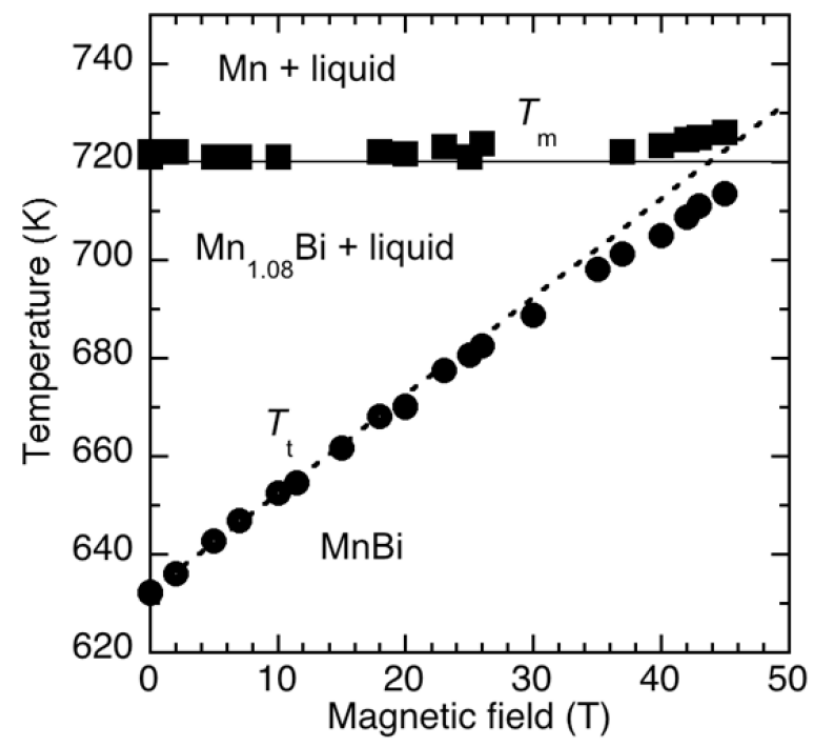

Pис. 3. Фрагмент фазовой диаграммы $\mathrm{MnBi}$ в магнитном поле. Пунктирная линия - экстраполяция, рассчитанная с использованием метода наименьших квадратов с данными для $B \leq 14$ Тл [29].

Fig. 3. Fragment of phase diagram MnBi in magnetic field. The dashed line is an extrapolation calculated using the least squares method with data for $B \leq 14 \mathrm{~T}$ [29]. 
Эти же авторы провели расчет энергии магнитной анизотропии и заключили, что ее величина слишком мала для внесения каких-либо изменений в формирующуюся структуру данного сплава [36]. В то же время, авторы работы [33] утверждают, что увеличение текстурной компоненты $\{100\}$ в магнитоотожженных образцах Армко-железа обусловлено разницей в магнитной свободной энергии и её вкладом в полную энергию системы в условиях магнитного отжига, поскольку максимум снижения энергии Гиббса в магнитном поле происходит при образовании $\{100\}$ зародышей из деформационной структуры.

Наиболее часто объектом исследований влияния магнитного поля на структурообразование служат сплавы $\mathrm{Fe}-\mathrm{Si}$, ввиду своего широкого промышленного применения и текстурозависимых функциональных характеристик. Цикл работ [37-39] посвящён изучению влияния магнитного поля с индукцией до 19 Тл на формирование текстуры и тип образующихся границ в ходе первичной рекристаллизации неориентированной стали $\mathrm{Fe}-\mathrm{Si}$ с содержанием кремния 0,75 и 3,25\%. Показано, что сильное магнитное поле увеличивает объемную долю зёрен Госсовской ориентировки $\{110\}<001>$ в многокомпонентной текстуре рекристаллизации. Отмечается, что магнитное поле может замедлять формирование зародышей рекристаллизации, но ускоряет перемещение границ зерен. В более сильных полях эффект замедления компенсируется движущей силой магнитного поля, которая приводит к увеличению скорости миграции границ тех зерен, которые определенным образом ориентированы по отношению к полю. В [39] показано, что магнитное поле 17 Тл, приложенное при температуре отжига $800^{\circ} \mathrm{C}$ не увеличивает ориентационную плотность Госсовских зёрен и зёрен $\{100\}<001>$, однако увеличивает плотность аксиальной ориентировки $<110>\| \mathrm{RD}$ и уменьшает плотность аксиальной ориентировки $\gamma(<111>\| \mathrm{ND})$.

Влияние магнитного поля до 30 Тл на формирование структуры сплава $\mathrm{Fe}-1 \% \mathrm{Si}$ оценивали в работе [40]. Прикладывали магнитное поле 1.5 Тл, 15 Тл и 30 Тл в процессе отжига $\mathrm{Fe}-1 \% \mathrm{Si}$ образцов при $787^{\circ} \mathrm{C}$ (выше температуры Кюри на $27^{\circ} \mathrm{C}$ ) в течение 1 часа. Обнаруже- но усиление Госсовской компоненты в текстуре с увеличением поля, сопровождающееся значительным ростом зёрен. Предполагается, что магнитное поле ускоряет рост зерен в этих условиях. В более поздней работе [34] авторы обнаружили, что в образцах из сплава $\mathrm{Fe}-2,6 \% \mathrm{Si}$ после отжига в магнитном поле 7 и 14 Тл усиливается текстурная компонента $(<100>\| \mathrm{RD})$, а так же улучшаются магнитные свойства.

В работах [41-43] за счёт подбора температурных режимов отжига показано, что магнитное поле оказывает тормозящее воздействие на процессы возврата в холоднокатаных сплавах $\mathrm{Fe}-1,5(3) \% \mathrm{Si}$ и $\mathrm{Fe}-50 \% \mathrm{Ni}$. Это объясняется, в первую очередь, уменьшением диффузионной подвижности атомов в ферромагнитных сплавах при приложении внешнего магнитного поля, о котором сообщалось в $[30,44]$. В результате этого процессы перераспределения и аннигиляции дислокаций, за счёт которых и происходит разупрочнение, при наложении магнитного поля протекают медленнее. Альтернативное объяснение, представленное в [41], основано на том, что наличие большого количества дислокаций оказывает разориентирующее действие на спиновый порядок в ферромагнетиках, [45-47]. Поэтому перемещение дислокаций в объеме деформированного материала при наложении магнитного поля замедляется из-за появления дополнительной энергии, которую необходимо затратить для преодоления дислокациями ориентирующего влияния поля [45].

Кроме того, показано, что изменения, вносимые магнитным полем в процесс формирования кристаллографической текстуры, начинаются уже на стадии дорекристаллизационного отжига [42], что обнаруживается затем при последующей рекристаллизации уже без магнитного поля (Рис. 4) [44].

Это позволяет утверждать, что влияние магнитного поля на формирование текстуры не связано с изменением особенностей движения большеугловых границ на стадии первичной рекристаллизации, о чем говорилось, например в [39], а причиной влияния магнитного поля на формирование текстуры является зависимость магнитной части свободной энергии от угла между кристаллографическими осями и магнитным полем.
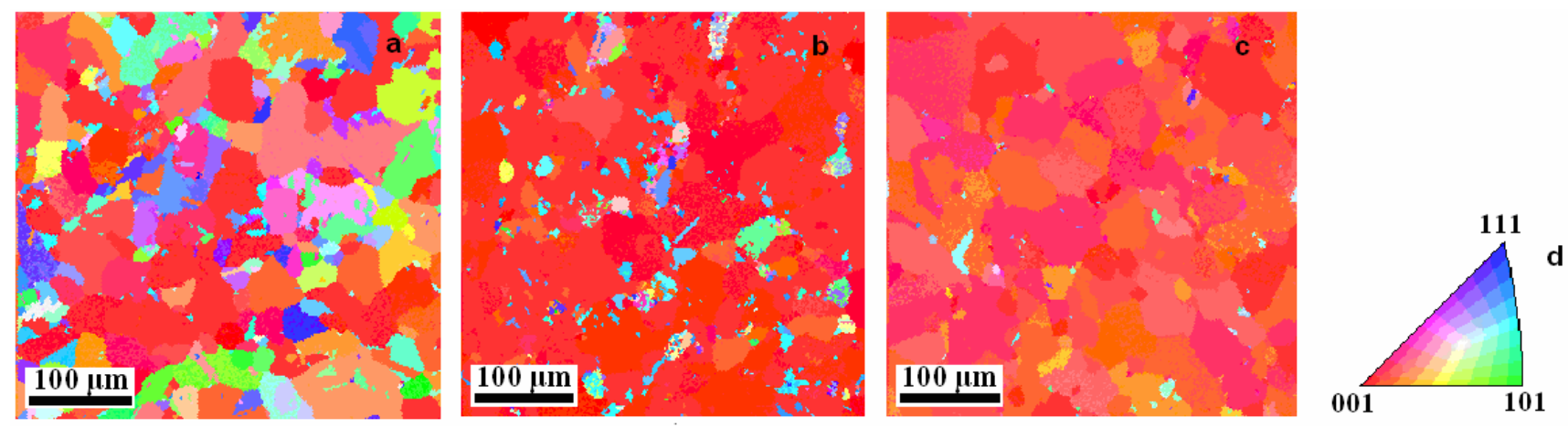

Pис. 4. (Color online) Ориентационные карты (a-c), и стереографический треугольник с расшифровкой ориентаций (d) образцов из сплава $\mathrm{Fe}-50 \% \mathrm{Ni}$; после медленного нагрева до $675^{\circ} \mathrm{C}$, предварительно отожженных при $500^{\circ} \mathrm{C}$ без поля (a), в поле 20 Тл (b) и 29 Тл (с) [42].

Fig. 4. (Color online) Orientational maps (a - c) and stereographic triangle with identified orientations (d) of the $\mathrm{Fe}-50 \% \mathrm{Ni}$ alloy after slow heating to $675^{\circ} \mathrm{C}$ and preliminary prerecrystallization annealing at $500^{\circ} \mathrm{C}$ without field (a), in a field of $20 \mathrm{~T}$ (b) and $29 \mathrm{~T}$ (c) [42]. 


\section{4. Заключение}

Приложение магнитного поля в процессе фазовых и структурных превращений является перспективным методом воздействия на конечную структуру металлических материалов. На сегодняшний день хорошо проработана термодинамика фазовых превращений в условиях сильного магнитного поля, Объяснение происходящих явлений при фазовых превращениях хорошо описывается в рамках термодинамических подходов, однако остаются открытыми вопросы относительно изменения кинетики таких превращений. До конца не установлены механизмы влияния магнитного поля на структурные превращения в холоднокатаных ферромагнитных сплавах. Это связано, в первую очередь с недостатком экспериментальных данных. Для продвижения в понимании природы наблюдаемых эффектов необходимы эксперименты на материалах с разными характеристиками, такими как величина магнитострикции, направление легкого намагничивания, температурные области протекания превращений. Помимо этого необходимо варьирование режимов отжига в магнитном поле для того, чтобы оценить влияние поля на отдельные стадии структурной эволюции.

Благодарность/Acknowledgements. Работа выполнена в рамках государственного задания ФАНО России «Магнит» № AAAA-A18-118020290129-5./The research was carried out within the state assignment of FASO of Russia (theme “Magnet" № AAAA-A18-118020290129-5).

\section{Литература/References}

1. I. Skorvánek, J. Marcin, M. Capik, M. Varga, J. Turcanová, J. Kovác, P. Svec, D. Janickovic, F. Kovác, V. Stoyka. Magnetohidrodynamics. 48, 371 - 377 (2012).

2. Patent RF № 2430975/28, 10.10.2011 (in Russian) [Патент РФ№ 2430975/28, 10.10.2011.

3. L. Neel, J. Phys. Radiat. 15, 225 (1954).

4. P. Kapitza. Transactions of the Oxford University Junior Scientific Club. 5, 129-133 (1931).

5. M. Yamaguchi, Y. Tanimoto. Magneto-Science: Magnetic Field Effects on Materials: Fundamental and Applications. Japan: Kodansha-Springer. (2006) 343 p.

6. U. Kolm, A. Freeman. Scientific American. 212, 66 (1965).

7. M. Enomoto. Materials Transactions. 46, 1088-1092 (2005).

8. Y. Zhang, C. Esling, X. Zhao, L.Zuo. Materials Science Forum. 638-642, 202-207 (2010). DOI: 10.4028/ www.scientific.net/MSF.638-642.202

9. E. A. Fokina, L. V. Smirnov, V.D. Sadovskiy. Fizika metallov I metallovedenie. 19, $592-595$ (1965) (in Russian) [Е. А. Фокина, Л. В. Смирнов, В. Д. Садовский. ФМM. 19, 592 - 595 (1965)]

10. T. Kakeshita, K. Shimizu. The Japan Institute of Metals. 11, $230-235$ (1986).

11. V.D. Sadovskiy, L. V. Smirnov, E. A. Fokina, P. A. Malinen, I. P.Sorokin. Fizika metallovImetallovedenie. 24,918-939 (1967) (in Russian) [В.Д. Садовский, Л.В. Смирнов,
Е. А. Фокина, П.А. Малинен, И.П. Сорокин. ФММ. 24,. $918-939$ (1967)]

12. I. Ya. Georgieva, O.P. Maksimova, P.A. Malinen, L.A. Melnikov, V.D. Sadovskiy. Fizika metallov I metallovedenie. 35, 363-369 (1973) (in Russian) [И.Я. Георгиева, О. П, Максимова, П.А. Малинен, Л. А. Мельников, В. Д. Садовский. ФММ. 35, 363 - 369 (1973)]

13. A. A. Leontyev, V.M. Schastlivtsev, L. N. Romashev. Fizika metallov I metallovedenie. 62, 138-144. (1986) (in Russian) [A.А. Леонтьев, В.М. Счастливцев, Л.Н. Ромашев. ФММ. 62, 138 - 144. (1986)]

14. V.D. Sadovskiy N.M. Rodigin, L.V. Smirnov, G.M. Filonchik, G. Fakidov. Fizika metallov I metallovedenie. 12, 302-304 (1961) (in Russian) [В.Д. Садовский, Н.М. Родигин, Л.В. Смирнов, Г. М. Филончик, Г. Факидов. ФММ. 12, 302 - 304 (1961)].

15. M.A. Krivoglaz, V.D. Sadovskiy. Fizika metallov I metallovedenie. 18, 502-505 (1964) (in Russian) [М. А. Кривоглаз, В. Д. Садовский. ФММ. 18, 502 - 505 (1964)]

16. V.M. Schastlivtsev, D.A. Mirzaev, Yu. V. Kaletina, E. A. Fokina. Physics of the solid state. 58, 336-345 (2016).

17. A.Z. Menshikov. Fizika metallov I metallovedenie. 28, $1012-1017$ (1993) (in Russian) [А.3. Меньшиков. ФММ. 28, 1012 - 1017 (1993)].

18. V.M. Schastlivtsev, Yu. V. Kaletina, E.A. Fokina, D. A. Mirzaev. Metal Science and Heat Treatment. 58, 247 - 253 (2016). DOI: 10.1007/s11041-016-9997-4

19. V.M. Schastlivtsev, Yu. V. Kaletina, E.A. Fokina. Martensitic transformation under magnetic field. Ekaterinburg, UB RAS. (2007) 322 p. (in Russian) [В.М. Счастливцев, Ю.В. Калетина, Е.А. Фокина. Мартенситное превращение в магнитном поле. Екатеринбург, УрО РАН. 2007. 322 с.]

20. H.D. Joo, S. U. Kim, N.S. Shin, Y.M. Koo. Materials Letters. 43, 225 - 229 (2000).

21. J-K. Choi, H. Ohtsuka, Y. Xu and W-Y. Choo. Scripta Mater. 43, $221-226$ (2000).

22. H. Ohtsuka. Materials Transactions. 48, 2851-2854 (2007).

23. X. J. Hao, H. Ohtsuka. ISIJ International. 46, $1271-1273$ (2006).

24. Y. Mitsui, Y. Ikehara, K. Takahashi, S. Kimura, G. Miyamoto, T. Furuhara, K. Watanabe, K. Koyama // Journal of Alloys and Compounds. 632, 251 - 255 (2015). DOI: $10.1016 /$ j.jallcom.2015.01.129

25. T. Fukuda, M. Yuge, J. Lee, T. Terai, T. Kakeshita. ISIJ International. 46, 1267 - 1270 (2006).

26. M.C. Gao, T. A. Bennett, A.D. Rollet, D.E. Laughlin. J. Phys. D: Appl. Phys. 39, $2890-2896$ (2006). DOI: 10.1088/0022-3727/39/14/003

27. H. Ohtsuka. Sci. Technol. Adv. Mater. 9, 013004-1-013004-7 (2008). DOI: 10.1088/1468$6996 / 9 / 1 / 013004$

28. T. Garcin, S. Rivoirard, E. Beaugnon. J. Phys. D: Appl. Phys. 44, 015001 (2011). DOI: 10.1088/0022-3727/44/1/015001

29. Y. Mitsui, Y. Ikehara, K. Watanbe. Journal of the Korean Physical Society. 62, $1769-1772$ (2013). 
30. Y. Mitsui1, K. Koyama, K. Watanabe. Materials Transactions. 54, 242 - 245 (2013).

31. S. Nakamichi, S. Tsurekawa, Y. Morizono, T. Watanabe, M. Nishida, A. Chiba. J. Mater.Sci. 40, 3191 - 3198 (2005).

32. R. Smolukowski, R. W. Turner. J. Appl.Phys. 20, $745-747$ (1949).

33. H.O. Martikeinen, V.K. Lindroos. Scandivian J. Metallurgy. 10, 3 -8. (1981).

34. M.Z. Salih, M. Uhlarz, F. Pyczak, H.-G. Brokmeier, B. Weidenfeller, N. Al-hamdany, W. M. Gan, Z. Y. Zhong, N. Schell. Journal of Magnetism and Magnetic Materials. 381, 350 - 359 (2015). DOI: 10.1016/j.jmmm.2015.01.004

35. N. Masahashi, M. Matsuo, K. Watanabe. J. Mater. Res. 13, 457 - 461 (1998).

36. B. Sawyer, A. Smoluchowski. J. Appl.Phys. 28, 1069- 1070 (1957).

37. C. M.B. Bacaltchuk, G. A. Castello-Branco, H. Garmestani. Materials Science Forum. 495 - 497, 1165 - 1170 (2005).

38. C. M. B. Bacaltchuk, G. A. Castello-Branco, H. Garmestani. Ceramic Transactions. 200, 405-411 (2009)

39. G. A. Castello-Branco, J. N. Muller, C.M. B. Bacaltchuk. Materials Science Forum.758.113-117 (2013). DOI: 10.4028/www.scientific.net/MSF.758.113

40. T. A. Bennett, R. A. Jaramillo, D. E. Laughlin, J. B. Wilgen, R. Kisner, G. Mackiewicz-Ludtka, G.M. Ludtka, P.N. Kalu, A.D. Rollett. Solid State Phenomena. 105, 151-156 (2005). DOI: 10.4028/www.scientific.net/
SSP.105.151

41. I. V. Gervasyeva, E. Beaugnon, V.A. Milyutin, E. G. Volkova, D.P. Rodionov, Y.V. Khlebnikova, D. A. Shishkin. Physica B. 468 - 469, 66 - 71 (2015). DOI: 10.1016/j.physb.2015.04.016

42. I. V. Gervas'eva V. A. Milyutin, E. Beaugnon, E. G. Volkova, D. A. Shishkin. Physics of metals and metallography. 116, $162-169$ (2015) (in Russian) [И.В. Гервасьева, В.А. Милютин, Э. Бинон, Е.Г. Волкова, Д. А. Шишкин. ФММ. 116, $171-178]$. DOI: 10.1134/ S0031918X15020064

43. I. V. Gervas'eva V.A. Milyutin, E. Beaugnon, V.A. Kazantsev, Y.V. Khlebnikova, D.P. Rodionov. Technical physics. 61, $1668-1673$ (2016) (in Russian) [И.В. Гервасьева, В.А. Милютин, Э. Бинон, В.А. Казанцев, Ю.В. Хлебникова, Д.П. Родионов. Журнал технической физики. 86, 64 - 69 (2016)]. DOI: 10.1134/S1063784216110098

44. X. J. Liu, Y. Lu, Y.M. Fang, C.P. Wang. CALPHAD. 35, $66-71$ (2011). DOI: 10.1016/j.calphad.2010.12.004

45. T.V. Efimova, V.V. Polotnyuk. UkrainePhys.J. 26, 1149-1152 (1981) (in Russian) [T.В. Ефимова, В. В. Полотнюк. Украинский физический журнал. 26, $1149-1152(1981)]$

46. F. Vicena. Czech. J. Phys. 5, 480-501(1955)

47. A. Seeger, H. Kronmuller, H. Rieger R. Traubler. J.Appl. Phys. 35, 740-748 (1964). 